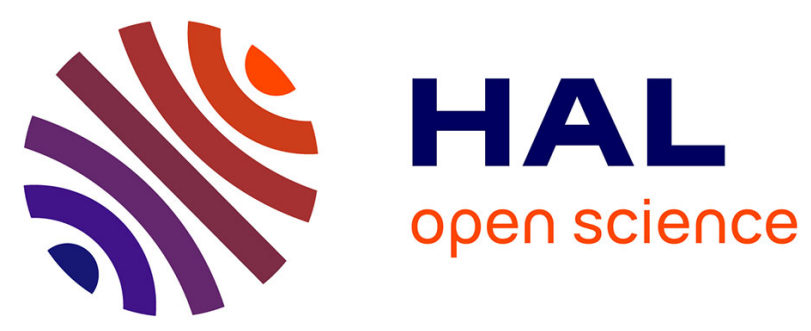

\title{
Steps Towards Quantification of the Cardiological Stress Exam
}

Radomir Chabiniok, Eva Sammut, Myrianthi Hadjicharalambous, Liya Asner, David Nordsletten, Reza Razavi, Nicolas Smith

\section{- To cite this version:}

Radomir Chabiniok, Eva Sammut, Myrianthi Hadjicharalambous, Liya Asner, David Nordsletten, et al.. Steps Towards Quantification of the Cardiological Stress Exam. Functional Imaging and Modeling of Heart, Jun 2015, Maastricht, Netherlands. pp.12-20, 10.1007/978-3-319-20309-6_2 . hal-01254914

\section{HAL Id: hal-01254914 \\ https://hal.science/hal-01254914}

Submitted on 12 Jan 2016

HAL is a multi-disciplinary open access archive for the deposit and dissemination of scientific research documents, whether they are published or not. The documents may come from teaching and research institutions in France or abroad, or from public or private research centers.
L'archive ouverte pluridisciplinaire HAL, est destinée au dépôt et à la diffusion de documents scientifiques de niveau recherche, publiés ou non, émanant des établissements d'enseignement et de recherche français ou étrangers, des laboratoires publics ou privés. 


\title{
Steps towards quantification of the cardiological stress exam
}

\author{
R. Chabiniok ${ }^{1,2}$, E. Sammut ${ }^{2}$, M. Hadjicharalambous ${ }^{2}$, L. Asner ${ }^{2}$, \\ D. Nordsletten ${ }^{2}$, R. Razavi ${ }^{2}$, and N. Smith ${ }^{2}$

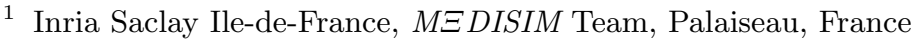 \\ 2 Division of Imaging Sciences \& Biomedical Engineering, St Thomas' Hospital, \\ King's College London, UK
}

\begin{abstract}
In this work we aim to advance the translation of modelbased myocardial contractility estimation to the clinical problem of quantitative assessment of the dobutamine stress exam. In particular, we address the question of limited spatial resolution of the observations obtained from cine MRI during the stress test, in which typically only a small number of cine MRI slices are acquired. Due to the relative risk during the dobutamine infusion, a safe acquisition protocol with a healthy volunteer under the infusion of a beta-blocker is applied in order to get a better insight into the contractility estimation using such a type of clinical data. The estimator is compared for three types of observations, namely the processed short axis cine stack contiguously covering the ventricles, the short axis stack limited to only 3 slices and the combination of 3 short and 3 long axis slices. A decrease of contractilities in AHA regions under the beta-blocker infusion was estimated for each observation. The corrected model (by using the estimated parameters) was then compared with the displacements extracted from 3D tagged MRI.
\end{abstract}

\section{Introduction}

A dobutamine stress test is used clinically to identify regional wall motion abnormalities to guide management on therapeutic options, such as revascularisation [7]. It is performed at increasing doses of dobutamine, an inotropic drug, with simultaneous imaging by echocardiography or magnetic resonance imaging (MRI) at each dosage level. Significant risks such as arrhythmia and cardiac arrest at higher doses, and the unpleasant sensation of the pharmacologically induced stress requires a rapid data acquisition which should not exceed three minutes at each dobutamine dosage. This limits the spatio-temporal resolution of the obtained image data. In particular in cardiac MRI - the modality used in this paper - a typical clinical protocol includes an acquisition of 3 slices in short axis placed in basal, mid- and apical third of left ventricle (LV), see Fig. 1 (middle), and 3 standard long axis images of LV (the so-called 2-, 3- and 4-chamber views). In addition to the limited spatial coverage, the demanding breath-holding under the dobutamine infusion often compromises the quality of image data.

A positive test is defined by a regional wall motion abnormality during stress and is currently clinically assessed entirely qualitatively. An estimation of some 

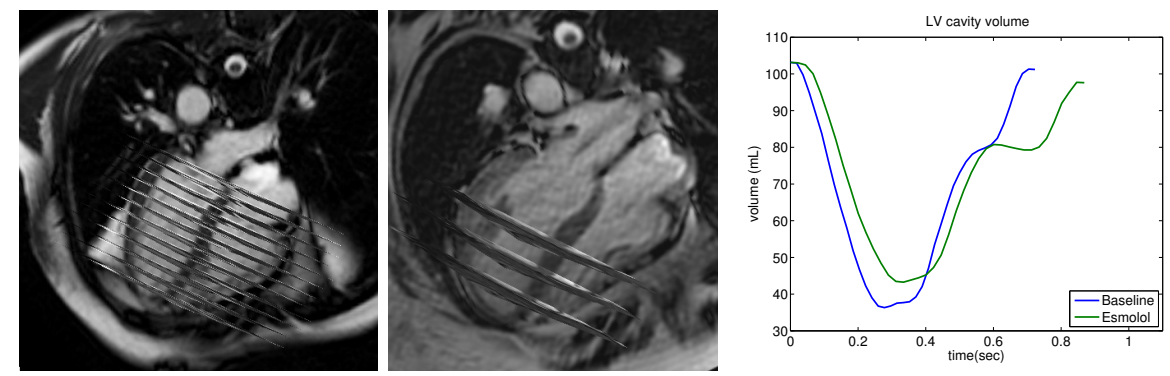

Fig. 1. Usual coverage of whole ventricles by short axis full cine stack (left) as oppose to the 3 short axis slices typically used in the stress exam (middle). The LV volume plot on the right demonstrates a decreased ejection during infusion of beta-blocker esmolol.

constitutive parameters in a biomechanical model is a way of assessing mechanical properties of myocardium $[4,13,12]$, and in [3] quantitative values directly targeting regional myocardial contractility were estimated using cine MRI as observations. Such an approach has a potential to provide a higher reproducibility of the exam, lower inter-observer variability and possibly may even allow a decrease in the dose of dobutamine as the quantitative measure may increase the sensitivity. However, in comparison to [3] - where a full short axis cine stack covering whole ventricles was used for contractility estimation - in the stress test we need to deal with the limited spatio-temporal coverage. The presented study focuses on exploring the feasibility of translating the methodology of [3] into the clinical setup of the dobutamine stress exam with respect to the challenge of limited coverage.

Due to the associated risks in using dobutamine, it would be impossible to perform trial acquisitions in a healthy volunteer or significantly prolong the patient exam to acquire additional short axis slices under the dobutamine stimulation. Therefore, to get an insight into various image sampling modes, we estimate a change of contractility in a healthy volunteer under the safe pharmacological stimulus of the beta-blocker esmolol. This allows safe acquisition of high quality image data of a full cine stack, which can be then downsampled in space or time, in accordance to the real clinical data of the dobutamine stress test. As opposed to the positively inotropic drug dobutamine, the negatively inotropic beta-blocker globally reduces the contractility. This is reflected in the decreased stroke volume (see Fig. 1, right) and we expect that the estimated contractility values should follow a similar trend.

After describing the experimental data together with image processing in Section 2, and the modeling and parameter estimation framework (Section 3), we present in Section 4 the results of contractility estimation at baseline and under beta-blocker stimulation by using processed cine MRI of various resolution. We discuss our results and give some perspective remarks in Section 5 and conclude the paper in Section 6 . 


\section{Clinical data}

\subsection{Data acquistion}

Cardiac MRI was performed on 1.5T Philips Achieva system. The acquisition of a healthy volunteer dataset was performed at baseline and under the infusion of beta-blocker esmolol (50-200 $\mu \mathrm{g} / \mathrm{kg} / \mathrm{min}$ to achieve 10-20\% decrease in heart rate) and the following data were acquired in each part of the study:

- Cine bSSFP sequence in retrospective ECG gating with spatio-temporal resolution 2x2x8mm and 40 time frames / cardiac cycle, FOV 350x350 mm. The short axis cine stack covering contiguously whole ventricles and standard long axis views of left ventricle (2-, 3-, and 4-chamber view) were taken.

- 3D tagged MRI of LV in prospective ECG triggering with acquired spatial resolution $3.4 \times 7.7 \times 7.7 \mathrm{~mm}$ (for 3 orientations of tag planes) reconstructed into one resulting $3 \mathrm{D}$ tagged image interpolated to $1 \mathrm{x} 1 \mathrm{x} 1 \mathrm{~mm}$ voxel size and temporal resolution $\sim 33 \mathrm{~ms}$ for both baseline and esmolol scan.

\subsection{Data processing}

Image processing consists first of spatial registration of all the image sequences (rigid registration using the Image Registration Toolkit IRTK ${ }^{3}$ ). Then, we select 3 slices from the short axis stack such that the inter-slice spacing corresponds to that one in real stress exam. Non-rigid image registration based motion tracking [11] is consequently employed to track the LV endo- and epicardial surfaces in cine MRI data. By motion tracking of various types (and combinations) of cine images, we generate three types of observations (deforming LV endo- and epicardial surfaces) which are used in sequel for the contractility estimation. These will be denoted as:

1. full s.a. stack: the short axis cine stack covering whole ventricles with the temporal resolution downsampled to 20 time frames / cardiac cycle;

2. 3-s.a.+3-l.a.: the selected 3 slices in short axis with 3 long axis views in original temporal sampling 40 time frames / cycle (standard clinical protocol);

3. 3-slice s.a.: the selected 3 slices of the full stack (40 time frames / cycle).

Remark 1. The temporal resolution for full s.a. stack used in the parameter estimation is such that the MRI acquisition time corresponds to the standard clinical protocol 3-s.a.+3-l.a.

Additionally, we extract full tissue displacements from 3D tagged MRI by using the tracking algorithm [11] tuned to the specific spatio-temporal resolution of the tagged data. The full displacements will not be used in this study as observations, however the error between the simulated and extracted displacement will be assessed. More details about image processing of such particular type of MRI dataset can be found in [2].

\footnotetext{
${ }^{3}$ http://www.doc.ic.ac.uk/ dr/software
} 
IV
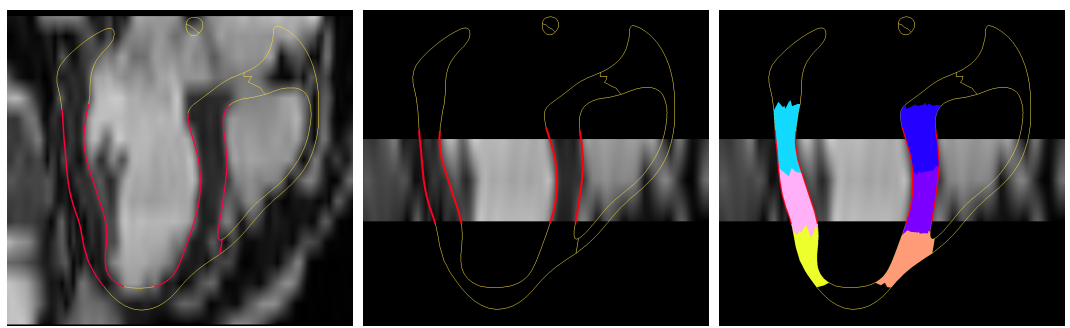

Fig. 2. From left to right: "pseudo 4-chamber" view reconstructed from full short axis cine stack consisting of $8 \mathrm{~mm}$-slices without gap with the contours of geometrical model; p4-ch view from 3-s.a. slices (inter-slice spacing 16mm); standard AHA segments. The red parts of LV endo- and epicaridal surfaces are used in the estimation to measure the data-model discrepancy.

\section{Modeling and parameter estimation framework}

The model and data-asimilation procedure used in this work are described in [5], [3], respectively, with more particular details of the estimator setup in [2]. In a nutshell, we use a continuum mechanics based model of biventricular anatomy. The model consists of an active contraction component (Bestel-Clément-Sorine model) and the passive tissue is represented by the visco-elastic material with the hyperelastic potential given by $W_{e}=\kappa_{1}\left(J_{1}-3\right)+\kappa_{2}\left(J_{2}-3\right)+\kappa(J-1-$ $\ln J)$ (Ciarlet-Geymonat). The model is activated by an analytically-prescribed physiological electrical activation wave.

The data-assimilation is based on reduced-order unscented Kalman filtering [8]. The discrepancy between the model and processed image data is evaluated by means of signed distances between the corresponding LV endo- and epicardial surfaces [10]. The parts of the cardiac surfaces on which the model-data discrepancy is evaluated is given by the coverage of the heart by the observations (see red surfaces in Fig. 2). Anatomically-created AHA regions are encompassed by the full s.a. and 3-s.a.+3-l.a. observation surfaces. In the 3-slice s.a. case the mid-cavity AHA segments are nearly fully encompassed by those surfaces, however the top half of the basal segments and the apical segments are not included in the imaging data (Fig. 2, right).

The model is pre-calibrated manually at the baseline so that the global volume indicators correspond to the clinical data. The Windkessel models representing the circulations are calibrated so that the simulated end-systolic and end-diastolic aortic pressures correspond to the measured values at baseline, and physiological values for the pulmonary circulation are assumed. This precalibration is used as an initialization for the contractility estimation both for the baseline and esmolol cases. The estimation of the contractility parameter is performed on the standard AHA subdivision for all 3 types of observations. 


\section{Results}

Fig. 3 (top) shows consistent estimates of myocardium contractility in AHA regions of a healthy volunteer at baseline using all types of observations. Under the beta-blocker stimulation, a decrease of contractility was detected in all segments when the full s.a. stack was used, and we can notice that the contractility decrease is lowest in the septal wall (particularly the apical septal part). When using the 3-s.a.+3-l.a. standard clinical protocol the drop of contractility is smaller but from a qualitative point of view it is similar to the full s.a. stack case - relatively homogeneous except for the septal wall. Finally, the 3-s.a. slices observations detect the lowest contractility drop and in particular "do not see" any contractility change in the regions not covered by the observation surfaces, i.e. the apical regions.
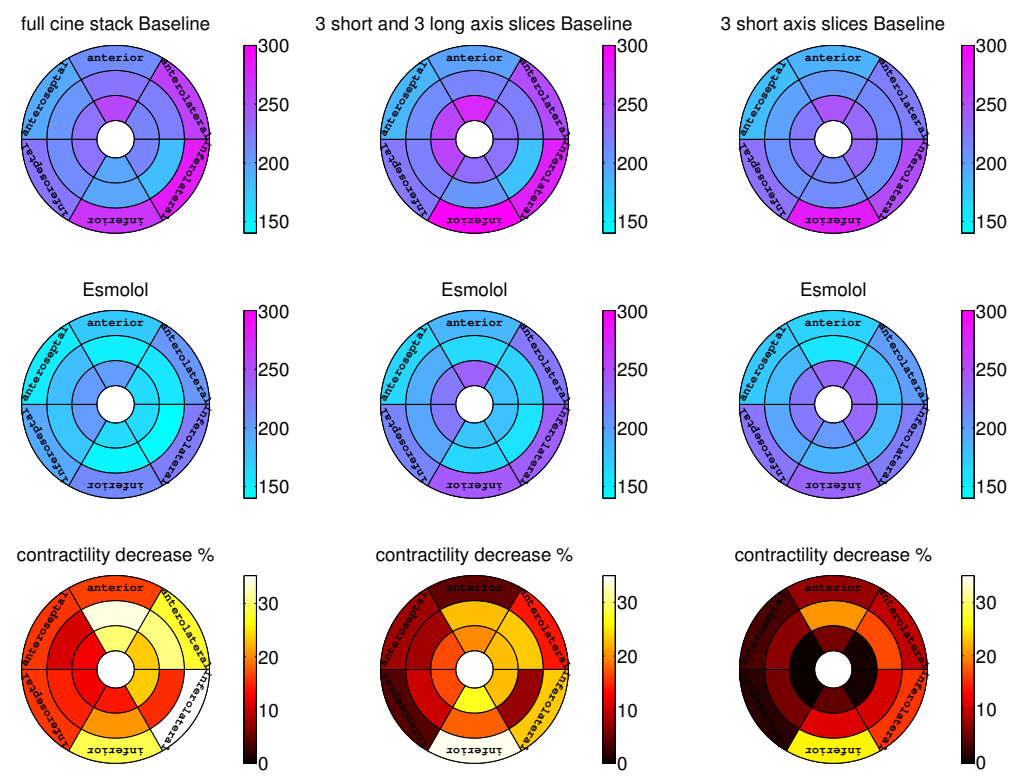

Fig. 3. Contractility (in $\mathrm{kPa}$ ) estimated using various type of observation surfaces at baseline (top) and during esmolol infusion (mid row); relative drop of contractility under the infusion of beta-blocker for each type of observations (bottom).

A global decrease of contractility is expected under the beta-blocker infusion in a healthy volunteer. However, the results from Fig. 3 cannot be compared to any ground truth as this is not available in vivo. To support our result, we employ the displacements extracted from the 3D tagged MRI and assess the error of radial component of displacement in each node of the tetrahedral mesh 
within the AHA regions over the systole and average it over the AHA region $i$ : error $_{i}=\frac{1}{\left|n \in A H A_{i}\right| \mid t \in \text { systole } \mid} \sum_{t \in \text { systole }} \sum_{\text {node }} \in \in A H A_{i}\left|\left(d_{n, \text { model }}^{t}-d_{n, 3 \text { Dtag }}^{t}\right) \cdot u_{n}^{r}\right|$, with $d$ denoting the incremental displacement between two consecutive time frames of tagged MRI and $u_{n}^{r}$ the vector of radial direction. Fig. 4 shows the improvement of this radial error for the esmolol case after inputting the estimated contractility parameters into the heart model with respect to the initial model parameterization.
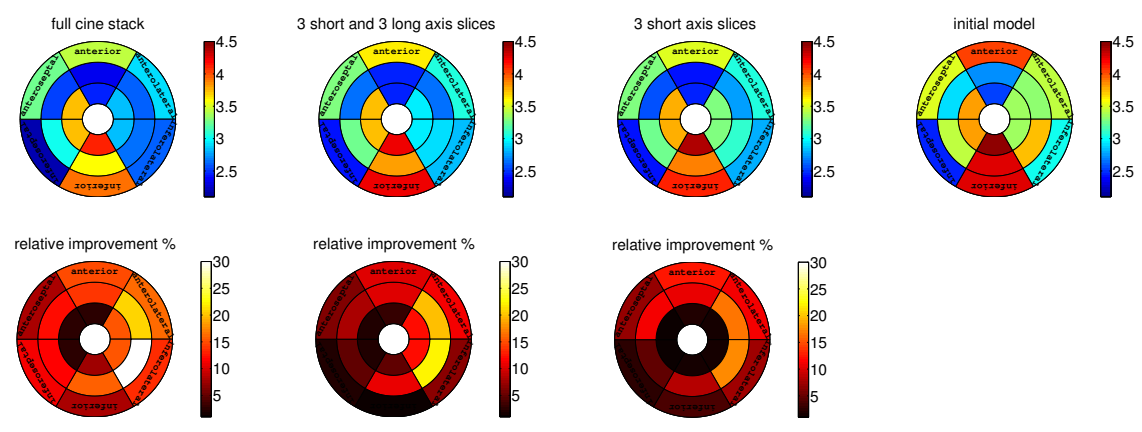

Fig. 4. Evaluation of radial error in the model (the initial parametrization and after the contractility estimation) with respect to radial displacements extracted from 3D tagged MRI: absolute error (top); relative drop of error per segment (bottom).

\section{Discussion}

The presented paper addresses a specific issue of applying model-based contractility estimation [3] in the quantification of dobutamine stress tests, used routinely to assess cardiac patients for consideration of revascularisation, namely the decreased spatial sampling of cine MRI. In this work we used esmolol, a negatively inotropic agent, to evaluate the optimal coverage and resolution of image data, and tested the whole data acquisition-processing-modeling and parameter estimation framework using a high quality volunteer dataset with pharmacologically modulated contractility.

As shown in Fig. 3 (top, estimation at baseline), if the model is sufficiently pre-calibrated by means of a low discrepancy with the observations, the estimator does not need to do much correction and the final regionally estimated parameters are consistent in between all types of observations. However, if the initialization is further apart from the data - as in our esmolol case - the estimator needs to correct more. As expected, the estimated quantities better correct the part of the model encompassed by the observation surfaces, and the regions further from those surfaces (e.g. apical region in the 3-s.a. observations) will 
be less sensitive and the estimator will perform worse. Evaluating Grammian matrix [9] would provide an insight into the sensitivity of parameters in each region, and will enrich our work in future.

In this study we have focused on detecting the decrease of regional contractility and on the associated correction of the model displacements. In the absence of ground truth, we evaluated the error with respect to the displacements extracted from the 3D tagged MRI - the image data not used in the modeling-parameter estimation framework applied in this paper.

Visually, a simple qualitative assessment of the patterns of relative contractility decrease and the relative error correction for the esmolol case (Figs. 3 and 4) supports the idea that the model is being corrected by decreasing the contractility. These figures show that in the septal segments (particularly the apical septal part) the estimator was not able to detect a contractility drop sufficiently and the error in the displacements in the model was not corrected. The apical septal regions may suffer either from a low quality of observations (suboptimal quality of segmentation) or from a more significant modeling error. Both may be caused by the complex geometry and fibre directions at the insertion place of RV. Adding the observations obtained by segmenting the RV surfaces might improve this issue.

In our tests, both the full cine stack and the 3-s.a.+3-l.a. protocol showed qualitatively similar decrease of contractility with esmolol. This suggests that the usual clinical protocol of 3-s.a.+3-l.a. could be as suitable for estimating the relative contractility change as the full s.a. cine stack. The 3-s.a.+3-l.a. protocol may however have two drawbacks: Firstly, the quantitative values of the detected contractility drop are significantly lower than if the full cine stack is used. Secondly, an important prerequisite for the motion tracking combining s.a. and $l . a$. views [11] is that those images are very well spatially aligned (to correct the non-reproducibility of patient's breath-holding). This spatial registration was efficient for our volunteer scan in which the full short axis cine stack provided sufficient information from the whole heart. When using patient data the spatial registration of the 3-s.a. slices with 3-l.a. slices would be a very challenging task.

The acquisition time for the full short axis cine stack in a spatio-temporal resolution as was used in our estimation trials is comparable to the acquisition of 3-s.a.+3-l.a. slices in standard temporal resolution. The presented work shows that a full-coverage short axis cine stack with a reduced temporal resolution may be an interesting alternative if quantification of results becomes a priority. We are aware that this hypothesis would need to be assessed by a study including a higher number of subjects, and it is one of our current targets.

We have calibrated the aortic pressure to the measured diastolic and systolic values at baseline, and we kept the pressure for the case with drug. It is reasoned by the fact that esmolol acts as a cardiac specific beta-blocker and therefore has a lower effect on the periphery circulation. Even if the pressure was not taken at each level of drug, the estimation would still provide a relative change in contractilities between the LV regions. Although we would be missing the scaling 
factor for the contractilities, the heterogeneity between the regions might provide the information to diagnose regional defects in contractility.

Long running times of the estimation (convergence of parameters) could be addressed by the preconditioning for instance by a reduced model [1] or statistical methods [2]. The state estimator [9] was not used in this work to allow for a higher sensitivity to the parameters, but would most likely accelerate the convergence. The optimal setup of the estimator is our ongoing work. A coarser subdivision e.g. into 6 radial regions may be considered when only a limited spatial coverage by images is available (e.g. the 3-s.a. slices in our trial). This result of estimation might be of a similar clinical value for a significantly lower computational cost.

We did not consider tagged MRI as observations, although it was shown that they are superior to cine MRI for estimating the tissue contractility [6]. In a very limited image acquisition time and the current practice of qualitative assessment of the dobutamine stress exam, it is unlikely that the tagged data would be acquired in near future in such an exam.

\section{Conclusion}

In this paper, we have investigated the types of data acquisition able to estimate a change of myocardial contractility in the clinical setup of dobutamine stress test. By carefully designing experimental data acquisition and adjusting the modeling-parameter estimation framework, the approach can provide an insight into the optimal imaging protocol to most accurately answer the clinical question. Simultaneously to the "computer-assisted diagnosis" we are therefore targeting the topic of "model-assisted optimal imaging".

The problem of estimating the contractilities in dobutamine stress tests has a number of unresolved subtasks and this work is providing initial steps to some of them, in particular the issue of coverage of myocardium by the image data, and the level of spatial discretization for the estimated parameters depending on the image data. Further development of the modeling and data-assimilation components, extending the number of subjects, challenging the image processing part by the image quality realistic in the dobutamine stress examinations and starting the estimation in the real dobutamine cases - with an accordingly modified scanning protocol - is a natural continuation of the presented work.

\section{Acknowledgments}

The authors acknowledge the support of Engineering and Physical Sciences Research Council EP/H046410/1, British heart foundation grant NH/11/5/29058 and Cardiovascular Healthcare Technology Cooperative. In addition, the author are thankful to P. Moireau and D. Chapelle (Inria, France) for providing the HeartLab software library, used in this work for all modeling and estimation computations, and to D. Rueckert and W. Shi (Imperial College London, Ixico) for providing the IRTK based motion tracking and valuable discussions. This research was supported by the National Institute for Health Research (NIHR) 
Biomedical Research Centre at Guy's and St Thomas' NHS Foundation Trust and King's College London. The views expressed are those of the author(s) and not necessarily those of the NHS, the NIHR or the Department of Health.

\section{References}

1. M. Caruel, R. Chabiniok, P. Moireau, Y. Lecarpentier, and D. Chapelle. Dimensional reductions of a cardiac model for effective validation and calibration. Biomech Model Mechanobiol, 13(4):897-914, 2014.

2. R. Chabiniok, K.K. Bhatia, A. King, D. Rueckert, and N. Smith. Manifold learning for cardiac modeling and estimation framework. In Proc. of 5th international STACOM workshop, pages 284-294. Springer Switzerland, 2015.

3. R. Chabiniok, P. Moireau, P.-F. Lesault, A. Rahmouni, J.-F. Deux, and D. Chapelle. Estimation of tissue contractility from cardiac cine-MRI using a biomechanical heart model. Biomech Model Mechanobiol, 11(5):609-30, 2012.

4. D. Chapelle, M. Fragu, V. Mallet, and P. Moireau. Fundamental principles of data assimilation underlying the Verdandi library: applications to biophysical model personalization within euHeart. Med Biol Eng Comput, 51(11):1221-1233, 2013.

5. D. Chapelle, P. Le Tallec, P. Moireau, and M. Sorine. An energy-preserving muscle tissue model: formulation and compatible discretizations. International Journal for Multiscale Computational Engineering, 10(2):189-211, 2012.

6. A. Imperiale, R. Chabiniok, P. Moireau, and D. Chapelle. Constitutive parameter estimation using tagged-MRI data. In Proc. of FIMH 2011, pages 304-312. Springer, 2011.

7. C. Jahnke, E. Nagel, R. Gebker, T. Kokocinski, S. Kelle, R. Manka, E. Fleck, and I. Paetsch. Prognostic value of cardiac magnetic resonance stress tests: Adenosine stress perfusion and dobutamine stress wall motion imaging. Circulation, 115:17691776, 2007.

8. P. Moireau and D. Chapelle. Reduced-order unscented Kalman filtering with application to parameter identification in large-dimensional systems. ESAIM: Control, Optimisation and Calculus of Variations, 17:380-405, 2011.

9. P. Moireau, D. Chapelle, and P. Le Tallec. Joint state and parameter estimation for distributed mechanical systems. Computer Methods in Applied Mechanics and Engineering, 197:659-677, 2008.

10. P. Moireau, D. Chapelle, and P. Le Tallec. Filtering for distributed mechanical systems using position measurements: Perspectives in medical imaging. Inverse Problems, 25(3):035010 (25pp), 2009. doi:10.1088/0266-5611/25/3/035010.

11. W. Shi, X. Zhuang, H. Wang, S. Duckett, D.V.N. Luong, C. Tobon-Gomez, K. Tung, P. Edwards, K. Rhode, R. Razavi, S. Ourselin, and D. Rueckert. A comprehensive cardiac motion estimation framework using both untagged and 3D tagged MR images based on non-rigid registration. IEEE Trans. Med. Imaging, 31(6):1263-1275, 2012.

12. V.Y. Wang, H.I. Lam, D.B. Ennis, B.R. Cowan, A.A. Young, and M.P. Nash. Modelling passive diastolic mechanics with quantitative MRI of cardiac structure and function. Med Image Anal, 13(5):773-784, 2009.

13. J. Xi, P. Lamata, S. Niederer, S. Land, W. Shi, X. Zhuang, S. Ourselin, S. Duckett, A. Shetty, C. Rinaldi, D. Rueckert, R. Razavi, and N Smith. The estimation of patient-specific cardiac diastolic functions from clinical measurements. Med Image Anal, 17(2):133-146, 2013. 